\title{
Particle Dynamics in Colloid-Polymer Mixtures with Different Polymer Architectures
}

\author{
Qimeng Wu, Ruben Higler, Thomas E. Kodger, and Jasper van der Gucht* \\ Cite This: ACS Appl. Mater. Interfaces 2020, 12, 42041-42047 \\ Read Online
}

ABSTRACT: Nonadsorbing polymers are widely used as thickening agents for colloids. A quantitative description of the structure and dynamics of such colloid-polymer mixtures is crucial to reveal the mechanisms accounting for the desired mechanical properties. We use confocal microscopy to study colloids with three types of commonly used polymers with different architectures: linear, subgranular cross-linked, and branched microgels. All three thickeners give rise to heterogeneous colloidal dynamics, characterized by non-Gaussian displacement distributions. However, while the ensembleaveraged particle dynamics in these materials are very similar, the underlying individual particle dynamics are not. Linear polymers give rise to depletion attraction and the formation of colloidal gels, in which the majority of particles are immobilized, while a few weakly bound particles have much higher mobility. By contrast, the branched and cross-linked polymers
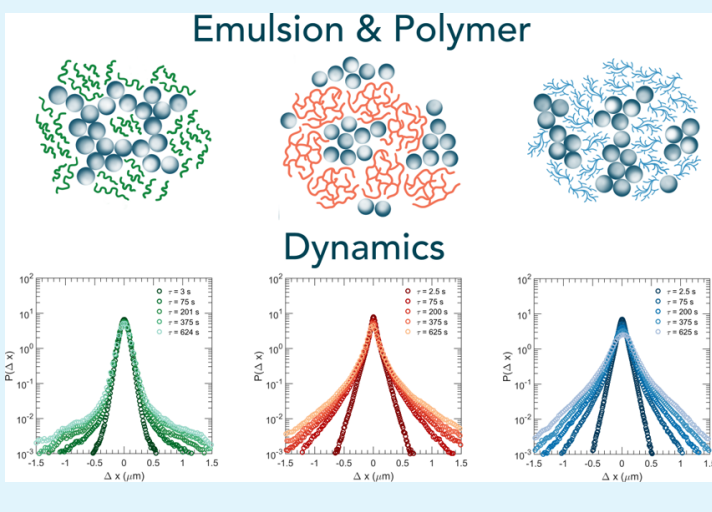
Dynamics thicken the continuous phase of the colloid, squeezing the particles into dense pockets, where the mobility is reduced and requires more cooperative rearrangements.

KEYWORDS: colloid composite, additives, polymer architecture, connectivity, step-size distribution

\section{INTRODUCTION}

Colloids exhibit rich rheological behavior ranging from lowviscosity fluids to elastic solids, depending on the volume fraction of particles, $\phi$, and the interaction between the particles. ${ }^{1,2}$ A soluble polymer is often added to the solvent to elicit an interaction between particles which modifies the mechanical properties, for example, by forming an elastic colloidal network at low volume fractions of particles. ${ }^{3}$ Alternatively, incorporation of thickening polymers, such as guar and xanthan gum, can change the mechanical properties of the mixture by thickening the continuous phase, presumably without altering the interparticle interaction. ${ }^{4,5}$ Such colloidpolymer mixtures are widely encountered in, for instance, food products, cosmetics, coatings, and drug delivery systems, where their mechanical stability determines the shelf life of the products and their performance during use. ${ }^{6,7}$

The resulting complex viscoelasticity of colloid-polymer mixtures is intimately coupled with their microstructure and internal dynamics. ${ }^{8}$ Therefore, quantitative descriptions of the microstructure and dynamics of such materials are crucial to reveal the mechanisms accounting for the desired mechanical properties. Most experimental, ${ }^{9-11}$ simulation, ${ }^{12-15}$ and theoretical studies have focused on systems with attractive interactions between particles because of depletion attraction induced by a nonadsorbing polymer or depletion-like Morse potential. Attraction mediates the formation of samplespanning clusters, which dynamically arrest to create a gel which is heterogeneous in local connectivity, mesoscopic structure, and dynamics. ${ }^{16-20}$ Such colloidal gels derive their rigidity from physically bonded particles which form strands and connecting nodes that develop into a percolating elastic network. $^{21,22}$ Restructuring these networks due to external or internal stresses is governed by events happening at the individual particle level, as the bonds between the particles are typically weak; a relation between local connectivity and thermally activated dynamics at the individual particle level has been reported recently for a depletion-induced colloidal gel. $^{23,24}$

By contrast, local particle dynamics within mixtures, where polymers act as thickeners of the continuous phase, have received far less attention. Previous work on polymer melts containing hard nanoparticles, which have been studied extensively because of their technological importance in batteries, sensors, and implants, ${ }^{25}$ has shown that the rheological properties of such composite materials depend very sensitively on the polymer-particle and particle-particle interactions. $^{26,27}$ Also, well studied are mixtures of rigid

Received: April 18, 2020

Accepted: August 19, 2020

Published: August 19, 2020 

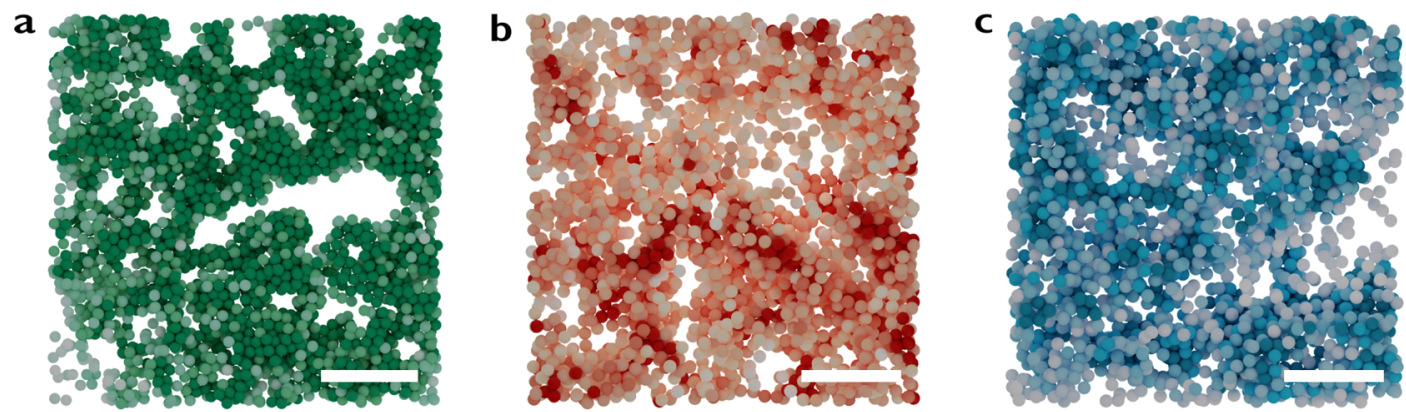

Figure 1. Computer-generated renderings from experimentally determined particle positions for (a) PVP, $c_{\mathrm{p}}=8.5 \mathrm{mg} / \mathrm{mL}$; (b) Carbopol, $c_{\mathrm{p}}=8.8$ $\mathrm{mg} / \mathrm{mL}$; and (c) PAA, $c_{\mathrm{p}}=6.6 \mathrm{mg} / \mathrm{mL}$ at a constant particle volume fraction, $\phi=0.25$. Particles are color coded based on their coordination number, $Z$. Increasing shades of the corresponding colors indicate increasing $Z$. Scale bar in all renderings is $15 \mu \mathrm{m}$.
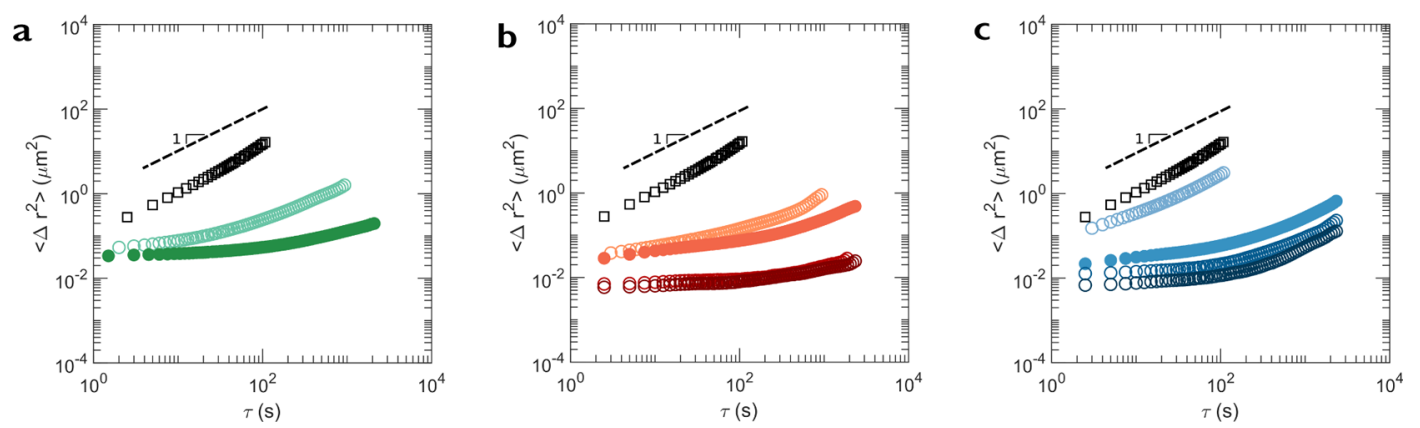

Figure 2. Ensemble-averaged MSD $\left\langle\Delta r^{2}\right\rangle$ of particles mixed with (a) PVP, $c_{\mathrm{p}}=0,6.5$, and $8.5 \mathrm{mg} / \mathrm{mL}$; (b) Carbopol, $c_{\mathrm{p}}=0,6.6,8.8,16.5$, and 19.8 $\mathrm{mg} / \mathrm{mL}$; and (c) PAA, $c_{\mathrm{p}}=0,4.4,6.6,8.8$, and $12.1 \mathrm{mg} / \mathrm{mL}$. The ensemble-averaged mean-squared displacements (MSDs) decrease with increasing polymer concentration. The data indicated with closed symbols in $(\mathrm{a}-\mathrm{c})$ correspond to samples with very similar $\left\langle\Delta r^{2}\right\rangle$ and are selected for a detailed comparison.

colloidal particles with soft particles, such as microgels ${ }^{28}$ or star polymers. $^{29,30}$ The difference in softness between the two types of particles results in distinctly unique interactions between the components, eliciting a diverse set of states both glassy and phase-separated, intrinsically relying on the penetrability of the constituent particles. ${ }^{31-33}$

While these studies clearly show that the dynamics in colloid-polymer mixtures depend strongly on the interactions between the different components and on their relative size and softness, a quantitative comparison between particle dynamics of colloids mixed with depletants and thickeners is not clearly established, hindering the tunability of mechanical properties in such colloid-polymer systems. The strong heterogeneity in particle dynamics in these systems makes a detailed direct comparison even more important because apparent similarities at the macroscopic level may have very different microscopic origins. Clearly, such a comparative study requires detailed observations at the individual particle level.

Additionally, nearly all studies on individual particle dynamics have been conducted on colloids containing rigid particles, while colloids comprising droplets remain largely unexplored. Not only do the droplets experience different intrinsic physics compared to rigid particles, for instance, surface mobility, deformation, and coalescence, they are also ubiquitously present in food products. ${ }^{34-37}$

In this paper, we study refractive index and density-matched colloid-polymer mixtures which consist of emulsions mixed with linear polymers, subgranular cross-linked polymers, and branched microgels. We use quantitative three-dimensional (3D) confocal microscopy to experimentally probe the microstructure and local dynamics of droplets at the individual-particle level. Our results show that for polymer concentrations where the ensemble dynamics of these materials are the same, the distribution of individual particle dynamics is dissimilar. The linear polymer gives rise to depletion gels, in which most of the particles are immobilized, while few, weakly bound, particles can make large excursions. By contrast, the branched and cross-linked polymers thicken the continuous phase and squeeze the particles into dense pockets, where particle mobility is characterized by caging and intercage hopping.

\section{RESULTS AND DISCUSSION}

Global Structure and Dynamics. We study monodispersed colloidal copolymer droplets with radius, $a=1.0 \mu \mathrm{m}$, made of poly(butyl acrylate)-poly(methyl acrylate) (PBAPMA), in a solution that closely matches the density of the particles; this allows the study of the structure and dynamics of the system in the absence of gravitational stresses. The addition of a low molar fraction of PMA to the predominate PBA particles allows matching the particle refractive index to the solution, minimizing scattering, and enabling $3 \mathrm{D}$ confocal microscopy. PBA has a low glass-transition temperature, $T_{\mathrm{g}}=$ $220 \mathrm{~K}$ and therefore exists as a viscous liquid at room temperature, so that the dispersion is an emulsion. These colloids are separately mixed with three types of polymers, a linear polyvinylpyrrolidone (PVP) K60, a subgranular crosslinked polymer (Carbopol), and a lightly cross-linked poly(acrylic acid) (PAA) polymer microgel, resulting in three distinct colloid-polymer samples, all with a constant volume fraction of droplets, $\phi=0.25$.

To begin evaluating the microstructure, we determine the coordination number, $Z$, for each particle by identifying all of 

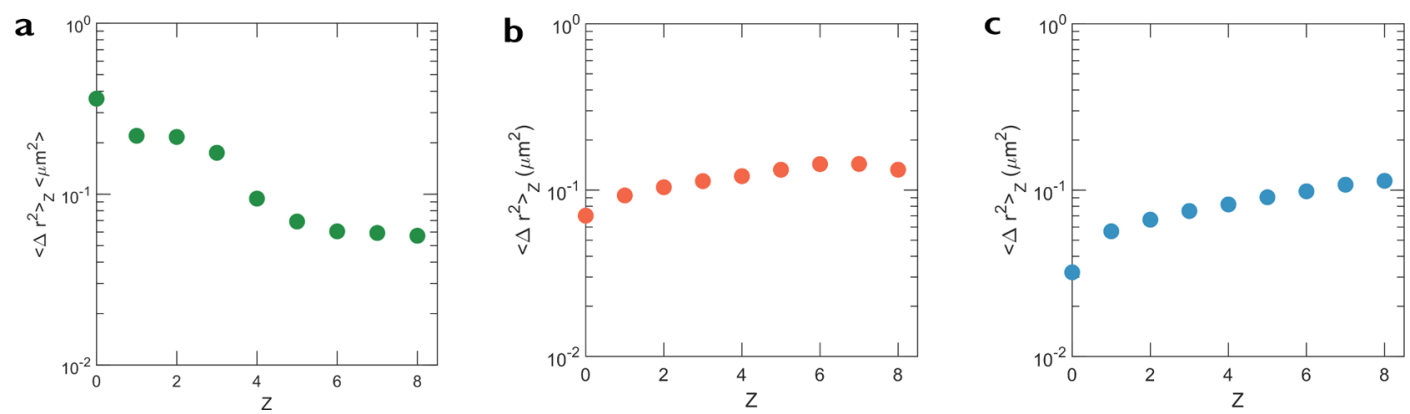

Figure 3. Particle MSDs $\left\langle\Delta r^{2}\right\rangle(Z)$ of particles mixed with (a) PVP, $c_{\mathrm{p}}=8.5 \mathrm{mg} / \mathrm{mL}$; (b) Carbopol, $c_{\mathrm{p}}=8.8 \mathrm{mg} / \mathrm{mL}$; and (c) PAA, $c_{\mathrm{p}}=6.6 \mathrm{mg} / \mathrm{mL}$ at $\tau=200 \mathrm{~s}$ as a function of coordination number $Z$.

its nearest neighbors from 3D image stacks acquired by confocal fluorescence microscopy, from which the particle positions are determined using previously developed algorithms. ${ }^{38,39}$ Particles are identified as the nearest neighbors if their center-to-center distance is within a cutoff value, $2.2 a$, with $a$ being the particle radius. For all three polymer architectures, we observe that adding a polymer to the colloid leads to a dramatic change in the structure, from isolated particles in the absence of the polymer to a very heterogeneous structure consisting of larger clusters at high polymer concentrations, as shown in Figure 1, where the deepening color shade indicates increasing $Z{ }^{11}$ This change in structure is also reflected in the particle dynamics, as indicated by the ensemble-averaged MSD $\left\langle\Delta r^{2}\right\rangle$ shown in Figure 2 as a function of lag time $\tau$ for three different samples. In the absence of polymer, there is a diffusive response $\left\langle\Delta r^{2}\right\rangle \propto \tau$, while, unsurprisingly, for all three samples, $\left\langle\Delta r^{2}\right\rangle$ decreases strongly with increasing polymer concentration.

While the structure and ensemble-averaged dynamics are very similar for the three different samples, the underlying mechanisms that lead to this behavior are quite different. The linear polymer (PVP) induces a depletion attraction between the particles because of the small polymer to particle size ratio, $\xi=R_{\mathrm{g}} / a<0.1$, where $R_{\mathrm{g}}=21 \mathrm{~nm}$ is the polymer radius of gyration measured by static light scattering. ${ }^{40}$ This attraction leads to particle aggregation and the formation of a colloidal gel (Figure 1a), in which particle motion is strongly suppressed and becomes subdiffusive (Figure 2a). For samples containing Carbopol, which consists of particles having a typical radius larger than $10 \mu \mathrm{m}$ and are athermal, there is negligible depletion attraction; yet, there is a similar heterogeneous microstructure (Figure 1b). This is likely due to volume exclusion by Carbopol particles, which squeeze the droplets into pockets confined between the Carbopol particles. Within each pocket, there is an increase in the local volume fraction of the droplets, which is responsible for the lower particle mobility (Figure 2b). Lastly, PAA consists of branched microgel-like particles and may therefore contribute to both the depletion attraction between the droplets and volume exclusion, reducing $\left\langle\Delta r^{2}\right\rangle$, as seen in Figures $1 \mathrm{c}$ and $2 \mathrm{c}$.

Connectivity and Local Dynamics. In the above paragraphs, we have seen that the ensemble-averaged MSDs of the three samples are very similar, even if the underlying mechanisms may be very different. However, ensemble averaging may mask the effect of local structures on the dynamics of individual particles in the mixture. ${ }^{24}$ To further investigate this, we examine the relationship between local connectivity, that is, $Z$ and individual particle dynamics for samples with very similar $\left\langle\Delta r^{2}\right\rangle$ for these three different polymers; specifically, the samples labeled with closed symbols in Figure 2. We determine the value of $\left\langle\Delta r^{2}\right\rangle(Z, \tau)$ at lag time $\tau=200 \mathrm{~s}$ for all three samples and show this as a function of $Z$, measured in the first frame for each time period $\tau$, as seen in Figure 3. Here, we see a clear difference between the three samples. For the PVP sample, which forms a colloidal gel, there is a decrease of $\left\langle\Delta r^{2}\right\rangle(Z)$ with increasing $Z$; a similar trend has been reported for colloidal gels formed by rigid particles. ${ }^{24}$ The observed decrease in $\left\langle\Delta r^{2}\right\rangle(Z)$ with increasing $Z$ can be attributed to the fact that particles are less likely to escape from the gel strands because of thermally activated debonding events when there are more bonds per particle. ${ }^{24}$ Surprisingly, the particle mobility in our samples is higher (by about a factor of 4$)$ for the same $Z(Z \geq 4)$ at a smaller $\tau$, compared to the particle mobility found in colloidal gels formed by rigid particles, ${ }^{24}$ even though the attraction strength is 5 times larger in our system (see Figure S1) and our particles have a radius of $1 \mu \mathrm{m}$ and theirs is of $700 \mathrm{~nm}$. This difference might result from the difference in surface mobility between the droplets studied here and the rigid particles studied in literature. The liquidliquid interface between droplets may, even with an attractive interaction, still be highly mobile due to sliding, which does not require bond-breaking events. By contrast, motions of a rigid particle bound to a gel strand requires the breaking of bonds. As a result, when having the same number of bonds, even at higher attraction strength, soft particles may still be more mobile than rigid particles. Note that in both cases, the polymer concentrations are below $c^{*}$, thus any effects of elasticity arising from the polymer concentration are negligible.

Contrary to the PVP sample which exhibits a continuous decrease in $\left\langle\Delta r^{2}\right\rangle(Z)$, the Carbopol and PAA samples show a slight but continuous increase in $\left\langle\Delta r^{2}\right\rangle(Z)$, with $Z=0$ having the smallest $\left\langle\Delta r^{2}\right\rangle(Z)$, Figure 3b,c. Carbopol and PAA are cross-linked and, as a consequence, dispersions of Carbopol and PAA have a measurable elasticity at these concentrations even in the absence of particles, while the linear polymer solution is simply viscous. Indeed, we measure with rheology storage moduli $G^{\prime}=1 \mathrm{~Pa}$ and $G^{\prime}=2 \mathrm{~Pa}$ for Carbopol and PAA, respectively, as seen in Figure S3 in the Supporting Information. When particles are embedded in an elastic medium, the background elasticity greatly hinders particle displacements. In the Carbopol sample, in which there is negligible interparticle attraction, the particles which reside next to other particles may therefore be more mobile than the ones embedded in the elastic medium, as overcoming the background elasticity to displace costs more energy than pushing away or sliding next to nonbonded particles. Correspondingly, the lowest mobility is found when a particle is embedded in the Carbopol dispersion, isolated from other 

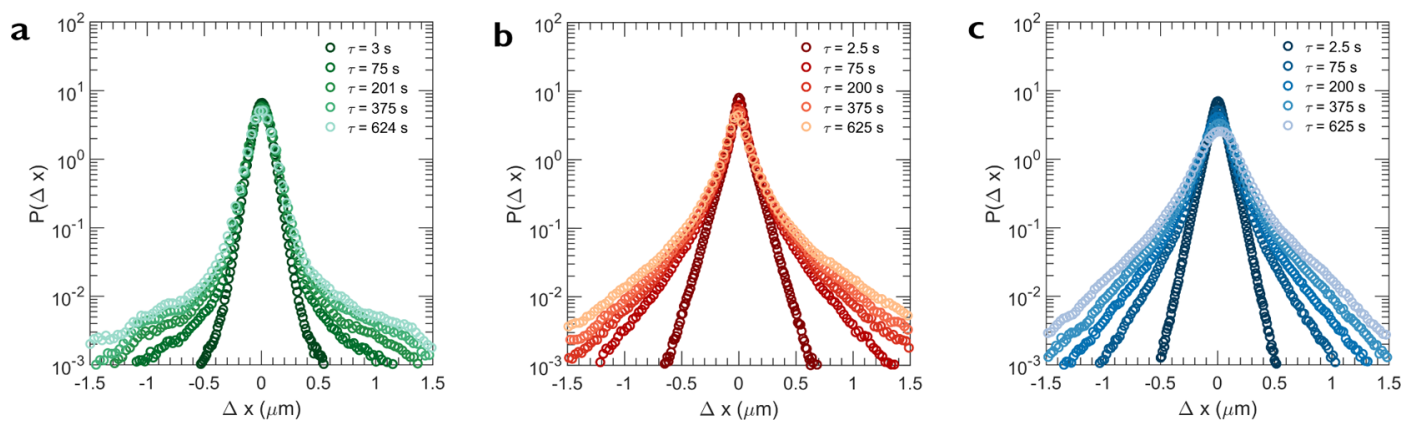

Figure 4. Time evolution of the self-part of the van Hove correlation function for particles mixed with (a) PVP, $c_{\mathrm{p}}=8.5 \mathrm{mg} / \mathrm{mL}$; (b) Carbopol, $c_{\mathrm{p}}=$ $8.8 \mathrm{mg} / \mathrm{mL}$; and (c) PAA, $c_{\mathrm{p}}=6.6 \mathrm{mg} / \mathrm{mL}$. Decreasing shades of the corresponding colors indicate an increase in the lag time, $\tau$.
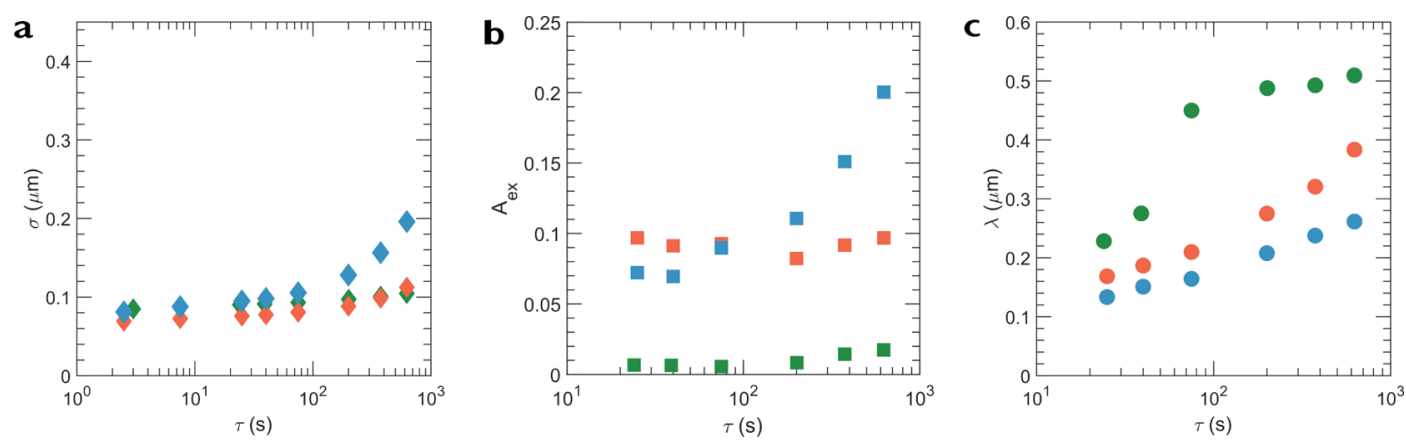

Figure 5. (a) Width of the Gaussian distribution $\sigma$. (b) Fraction of hopping particles $A_{\text {ex }}$ (c) Decay length of the exponential tail $\lambda$, for (green) PVP, $c_{\mathrm{p}}=8.5 \mathrm{mg} / \mathrm{mL}$; (orange) Carbopol, $c_{\mathrm{p}}=8.8 \mathrm{mg} / \mathrm{mL}$; and (blue) PAA, $c_{\mathrm{p}}=6.6 \mathrm{mg} / \mathrm{mL}$ as a function of lag time, $\tau$.

particles, $Z=0$, as seen in Figure 3b. PAA can induce both attraction between particles and background elasticity. We find that the behavior of the PAA sample is more similar to that of Carbopol, showing an increase in $\left\langle\Delta r^{2}\right\rangle(Z)$ with increasing $Z$. We thus conclude that the background elasticity has a larger effect on the mobility than the depletion attraction for the PAA sample.

Step-Size Distribution. Movies of each colloid-polymer sample show clear heterogeneous motions, see the Supporting Information for $2 \mathrm{D}$ movies. To further elucidate the microscopic dynamics at the individual-particle scale, we investigate the probability distribution of particle displacements, as characterized by the self-part of the van Hove correlation function. We consider only the $x$-component of the displacement and calculate the binned and normalized displacement probability distribution $P(\Delta x, \tau)$ by measuring the displacement $\Delta x(\tau)$ for each tracked particle. These distributions are shown for three different samples for different lag times in Figure 4; as seen in the symmetry of the distributions, the preshear and loading into sample chambers did not align or orient the sample microstructure. For all three types of polymers, we observe significant deviations from the Gaussian distribution expected for the Brownian motion. All distributions exhibit exponential tails, in addition to a central Gaussian region. Previous studies have shown that such non-Gaussian behavior is due to strongly heterogeneous particle dynamics, which can be qualitatively described as the superposition of two families of particles: localized particles, performing rattling motions around their initial positions, contributing to the Gaussian central part, and mobilized particles, performing sporadic larger displacements, or hops, leading to exponential tails. $^{41,42}$

We further analyze these data by extracting the width $\sigma$ of the central Gaussian region, the decay length $\lambda$ of the exponential tail, and the relative amplitudes of the two populations. We do this by fitting a Gaussian $P_{\mathrm{G}}(\Delta x)=\left(A_{\mathrm{G}} / \sqrt{2 \pi \sigma}\right) \exp \left(-\Delta x^{2} / 2 \sigma^{2}\right)$ to the central region $(|\Delta x|<0.3 \mu \mathrm{m})$ and an exponential function $P_{\mathrm{ex}}(\Delta x)=1 /$ $2 A_{\mathrm{ex}} \lambda \exp (-|\Delta x| / \lambda)$ to the tail region $(|\Delta x|>0.6 \mu \mathrm{m})$ (see Figure $\mathrm{S} 4$ in the Supporting Information). Here, $A_{\mathrm{G}}$ and $A_{\mathrm{ex}}$ denote the amplitudes of the Gaussian and exponential regions, which correspond to the fraction of localized and hopping particles, respectively.

In all three samples, the width of the Gaussian region $\sigma$ remains small and increases only slightly at very large $\tau$ (Figure 5a). This indicates that the particles belonging to this population are rattling around their average position and cannot move far. We note that the free self-diffusion time of these particles, $\tau_{\mathrm{B}} \approx 3 \pi \eta a^{3} / k_{\mathrm{B}} T$, is on the order of $1 \mathrm{~s}$, so that our data do not show the initial diffusive region expected for shorter times. The population belonging to the exponential tails, by contrast, shows a clear difference between the PVP sample, on the one hand, and the Carbopol and PAA samples on the other: in the PVP sample, $A_{\mathrm{ex}}$ is much smaller than in the other samples (Figure $5 b$ ), while $\lambda$ is much larger (Figure 5c). This indicates that this sample contains much fewer mobilized particles than the other two, but the typical distance that these mobilized particles move is much larger. This is likely related to the difference in the structure between the samples as the PVP sample is a colloidal gel and particles must detach from gel strands before they displace. Because of the attraction strength, $U \sim 7 k_{\mathrm{B}} T$, such debonding events are relatively rare, leading to a small population of mobile particles. However, the particles that do escape can explore different configurations in the gel before they reattach in a different location, so that the distance that they can travel is relatively large. By contrast, in the other two samples, the particles are 
confined to the interstitial spaces between the polymer particles, but there is negligible or much weaker attraction between the particles (see Supporting Information for calculation). The slow-down of the dynamics in this case is mostly due to an increase in the local volume fraction and may be compared with the dynamics in concentrated colloidal suspensions approaching a glass transition. ${ }^{43-45}$ It is well established that the dynamics in such systems is characterized by short-time rattling of particles within the cages formed by neighboring particles and occasional cage rearrangements, which involve a cooperative motion of multiple particles. These cage-breaking events give rise to dynamic heterogeneity and the non-Gaussian tails observed in the van Hove function. ${ }^{44}$ Apparently, these cage rearrangements occur more frequently than the debonding events in the colloidal gel sample. However, because of the high local particle volume fraction, they are limited in spatial extent, so that the typical distance, $\lambda$, moved by the mobile particles in these samples is smaller than that in the colloidal gel, as seen in Figure 5c.

These observations furthermore suggest that the samples with PAA microgels behave more like the Carbopol samples rather than like the PVP samples, indicating that the dominant effect of PAA is volume exclusion, rather than a depletion attraction. However, upon closer inspection, we do observe some differences between the PAA and Carbopol samples: in the PAA samples, the width of the Gaussian region gradually increases at large $\tau$, see Figures $4 \mathrm{c}$ and $5 \mathrm{a}$, indicating that the confinement in these samples is less severe than in the Carbopol samples. Moreover, the fraction of mobile particles increases as a function of $\tau$ in the PAA sample, while in the Carbopol sample, it remains more or less constant; this again implies a weaker confinement, as seen in Figure 5 b.

\section{CONCLUSIONS}

Our results demonstrate the necessity of dynamics at the individual-particle level in distinguishing materials consisting of colloids mixed with various architectures of polymers, which would help elucidate the origin of tunable viscoelastic behavior in such materials. In a colloidal gel, the dynamics are characterized by a large majority of immobilized particles and a small fraction of weakly bound particles that can make large excursions. By contrast, colloids that are thickened by subgranular polymer gel particles lead to dense pockets of particles characterized by heterogeneous dynamics reminiscent of concentrated colloidal suspensions or glasses. These microscopic differences could manifest macroscopically, for example, in how the materials age and coarsen, or in the longterm stability of products against syneresis or delayed failure. We hope to stimulate future theoretical efforts in understanding the interplay between particle dynamics and the complex rheological response in such colloidal composites. Additionally, the dependence of particle nature, rigid particle, or droplet on particle dynamics calls for interesting studies toward a complete description.

\section{EXPERIMENTAL SECTION}

Colloid-Polymer System. All chemicals are purchased from Sigma-Aldrich unless otherwise specified. We fabricate colloidpolymer mixtures consisting of PBA-MA particles, mixed with PVP K60 or PAA with two different architectures. PVP is chosen for these experiments to ensure a linear polymeric topology as the synthesis of PAA often leads to undesired branching and other side reactions during radical polymerization. ${ }^{46-49}$ Monodispered PBA-MA par- ticles, sterically stabilized by PVP K30, are synthesized according to a published method with modification. ${ }^{50}$ The recipe for seed particles by dispersion polymerization at $70{ }^{\circ} \mathrm{C}$ is as follows: a monomer mixture containing BA and MA with a ratio of $70 / 30$ (v/v \%); PVP $\mathrm{K} 30$ as a dispersant with $20 \mathrm{vol} \%$ of monomers; 4,4'-azobis-(4cyanopentanoic acid) as an initiator at $1 \mathrm{wt} \%$ to total monomer; and a solvent medium comprising methanol and $\mathrm{H}_{2} \mathrm{O}$ with a ratio of 88/ $12(\mathrm{v} / \mathrm{v} \%)$. PBA has a low glass-transition temperature $\left(T_{\mathrm{g}}\right)$ and thus exists as a liquid at room temperature. The droplets have a radius, $a=$ $1.0 \mu \mathrm{m}$, and a polydispersity of $\sim 5 \%$, as determined by optical microscopy and dynamic light scattering. The droplets are dispersed at a volume fraction $\phi=0.25$ in the medium of dimethyl sulfoxide (DMSO) and $\mathrm{H}_{2} \mathrm{O}$. This solvent mixture confers the ability to tune the solvent ratio to simultaneously match the refractive index and the density with that of the PBA-MA droplets. ${ }^{51}$ A ratio of $95 / 5$ (v/v \%) of DMSO and $\mathrm{H}_{2} \mathrm{O}$ is chosen for the current study with $3 \mathrm{mM} \mathrm{NaCl}$ to screen charge interactions. Droplets did not settle to any observable extent after centrifuging at $1200 \mathrm{~g}$ for $19 \mathrm{~h}$, confirming the neutral buoyancy of the samples. Optical clarity permits the samples to be observed with a confocal microscope approximately 100 $\mu \mathrm{m}$ into the sample without losing contrast. Sulfo-cyanine-3carboxylic acid (Lumiprobe) is dissolved in the continuous phase to enable fluorescence imaging through excitation by a $532 \mathrm{~nm}$ laser. PVP K60 has a molecular weight of $360,000 \mathrm{~g} / \mathrm{mol}$ with a radius of gyration $R_{\mathrm{g}}=21 \mathrm{~nm}^{40}$ and an estimated $c^{*}=3 M_{\mathrm{w}} / 4 \pi R_{\mathrm{g}}^{3} N_{\mathrm{A}}=15.4 \mathrm{mg} /$ $\mathrm{mL}$. The two different topologies of PAA are lightly cross-linked PAA $\left(M_{\mathrm{w}}=1,250,000 \mathrm{~g} / \mathrm{mol}\right)$ and Carbopol (Ultrez U10, Lubrizol) which is cross-linked PAA at the subgranular length scale. This allows us to have different size ratios between the droplets and polymers. A more detailed characterization of the added polymers is given in Table S1 in the Supporting Information, where the average size and the overlap concentration of the polymers are given. We also show a rheological characterization of the PAA and Carbopol samples, both with and without particles, in Figures S2 and S3 in the Supporting Information. We define the volume concentration of the polymer as the mass of the polymer, in milligrams, divided by the volume of the solvent, in milliliters. A range of concentrations of added polymers are investigated. Stock solutions of the colloids and the polymers are prepared separately and are used after a waiting period of at least $24 \mathrm{~h}$ to allow equilibration with the solvent mixture. Mixing of the samples is done by tumbling on a rotating wheel for $24 \mathrm{~h}$.

Confocal Microscopy and Image-Processing Analysis. Sample chambers are made of two rectangular cover slips $(21 \times 26$ $\mathrm{mm}$ ) glued to a standard glass microscope slide with a few $\mathrm{mm} 2 \mathrm{D}$ channel in-between. The sample area is approximately $5 \mathrm{~mm} \times 25$ $\mathrm{mm}$. A 3D channel is created by placing a third circular microscope cover glass (diameter $=50 \mathrm{~mm}$ ) on top with more glue. The empty chamber is exposed to ultraviolet light for the glue (Norland 61 NOA) to cure. The mixture of colloids and polymers is pipetted into the sample chamber, which is subsequently sealed using a twocomponent epoxy glue. 3D images are collected using a high-speed confocal microscope with a microlens array (VisiTech VT-Infinity3) and a silicon oil immersion objective (Nikon). The depth of the focal plane, $z$, is controlled by a piezoelement mounted on the microscope nosepiece. Images are captured at 780 or 1000 frames per second, with $256 \times 256$ pixels of size of $0.28 \mu \mathrm{m}$ in both the $x$-and $y$ directions. Each 3D image stack typically contains approximately 200 images, each offset by $0.2 \mu \mathrm{m}$ in the $z$-direction. One thousand stacks are obtained for all samples. Wall effects are avoided by capturing images at least $10 \mu \mathrm{m}$ away from the cover glass. Fluorescence is excited using a $532 \mathrm{~nm}$ DPSS laser; the fluorescence is filtered with a $560 \mathrm{~nm}$ long-pass filter and is detected with a Hamamatsu OCRA II CCD camera.

The intensity of the acquired images is inverted so that the particlerich phase appears bright and the particle-poor phase appears dark. First, a spatial band-pass filter is applied to the images to eliminate long-wavelength contrast gradients and short-wavelength pixel-topixel noise. Then, the Cartesian center of each droplet is located for each image stack with a previously developed algorithm. ${ }^{38,39}$ Based on the coordinates of the droplets, the quantitative 3D microstructure of 
the droplets can be rendered with a ray-tracing software (Blender). The coordination number for each droplet is determined by identifying all of its nearest neighbors. Droplets are identified as the nearest neighbors if their center-to-center distance is within a cut-off value, $2.2 a$. Once the coordinates have been found in each image stack, trajectories of droplets can be calculated, describing the droplet motion. ${ }^{38,39}$ The magnitude and the direction of the ensembleaveraged affine drift are computed after particles have been tracked. We then update particle locations by removing this drift, from which refined particle trajectories are calculated. We note that the drift was still apparent at a longer $\tau$; data at lag times for which the slope of the MSD versus $\tau$ is higher than 1, indicatingdrift, has been disregarded.

\section{ASSOCIATED CONTENT}

\section{SI Supporting Information}

The Supporting Information is available free of charge at https://pubs.acs.org/doi/10.1021/acsami.0c07153.

Attraction strength calculation; frequency sweep measurements; storage moduli of Carbopol and PAA as a function of added polymer concentration; characterization of the added polymer; and an example of the Gaussian fit and exponential fit for the step-size distribution (PDF)

PVP 2D $50 \mathrm{fps}$ (AVI)

Carbopol 2D 50 fps (AVI)

PAA 2D 50 fps (AVI)

\section{AUTHOR INFORMATION}

\section{Corresponding Author}

Jasper van der Gucht - Physical Chemistry and Soft Matter, Wageningen University and Research, Wageningen 6708WE,

The Netherlands; (1) orcid.org/0000-0001-5525-8322;

Email: jasper.vandergucht@wur.nl

\section{Authors}

Qimeng Wu - Physical Chemistry and Soft Matter, Wageningen University and Research, Wageningen 6708WE, The Netherlands

Ruben Higler - Physical Chemistry and Soft Matter, Wageningen University and Research, Wageningen 6708WE, The Netherlands; (1) orcid.org/0000-0002-4040-7898

Thomas E. Kodger - Physical Chemistry and Soft Matter, Wageningen University and Research, Wageningen 6708WE, The Netherlands

Complete contact information is available at:

https://pubs.acs.org/10.1021/acsami.0c07153

\section{Notes}

The authors declare no competing financial interest.

\section{ACKNOWLEDGMENTS}

This work was part of the Industrial Partnership Programme Hybrid Soft Materials that was carried out under an agreement between Unilever Research and Development B.V. and the Netherlands Organization for Scientific Research (NWO). The work of T.E.K. was part of a VENI funding scheme from NWO. The work of J.v.d.G. was part of the SOFTBREAK project funded by a Consolidator grant from the European Research Council.

\section{REFERENCES}

(1) Asakura, S.; Oosawa, F. On Interaction between Two Bodies Immersed in a Solution of Macromolecules. J. Chem. Phys. 1954, 22, $1255-1256$.

(2) Vrij, A. Polymers at Interfaces and the Interactions in Colloidal Dispersions. Pure Appl. Chem. 1976, 48, 471.

(3) Trappe, V.; Sandkühler, P. Colloidal gels - Low-density disordered solid-like states. Curr. Opin. Colloid Interface Sci. 2004, 8, 494-500.

(4) Phillips, G. O.; Williams, P. A., et al. Handbook of Hydrocolloids; CRC press: Boca Raton, FL, 2000.

(5) Dickinson, E. Stabilising Emulsion-based Colloidal Structures with Mixed Food Ingredients. J. Sci. Food Agric. 2013, 93, 710-721.

(6) Dickinson, E. Food Polymers, Gels and Colloids; Elsevier, 1991.

(7) Kreuter, J. Colloidal Drug Delivery Systems; CRC Press, 2014; Vol. 66.

(8) Larson, R. G. The Structure and Rheology of Complex Fluids; Oxford university press: New York, 1999; Vol. 150.

(9) Lekkerkerker, H. N. W.; Poon, W. C.-K.; Pusey, P. N.; Stroobants, A.; Warren, P. B. Phase Behaviour of Colloid Polymer Mixtures. Europhys. Lett. 1992, 20, 559-564.

(10) Ilett, S. M.; Orrock, A.; Poon, W. C. K.; Pusey, P. N. Phase Behavior of a Model Colloid-polymer Mixture. Phys. Rev. E 1995, 51, 1344-1352.

(11) Lu, P. J.; Conrad, J. C.; Wyss, H. M.; Schofield, A. B.; Weitz, D. A. Fluids of Clusters in Attractive Colloids. Phys. Rev. Lett. 2006, 96, $1-4$.

(12) Puertas, A. M.; Fuchs, M.; Cates, M. E. Comparative Simulation Study of Colloidal Gels and Glasses. Phys. Rev. Lett. 2002, 88, 098301.

(13) Colombo, J.; Gado, E. D. Stress localization, stiffening, and yielding in a model colloidal gel. J. Rheol. 2014, 58, 1089-1116.

(14) Landrum, B. J.; Russel, W. B.; Zia, R. N. Delayed yield in colloidal gels: Creep, flow, and re-entrant solid regimes. J. Rheol. 2016, 60, 783-807.

(15) Verweij, J. E.; Leermakers, F. A. M.; Sprakel, J.; van der Gucht, J. Plasticity in colloidal gel strands. Soft Matter 2019, 15, 6447-6454.

(16) Dinsmore, A. D.; Weitz, D. A. Direct Imaging of Threedimensional Structure and Topology of Colloidal Gels. J. Phys. Condens. Matter 2002, 14, 7581-7597.

(17) Prasad, V.; Semwogerere, D.; Weeks, E. R. Confocal Microscopy of Colloids. J. Phys.: Condens. Matter 2007, 19, 113102.

(18) Duri, A.; Cipelletti, L. Length Scale Dependence of Dynamical Heterogeneity in a Colloidal Fractal Gel. Europhys. Lett. 2006, 76, 972.

(19) Gao, Y.; Kilfoil, M. Direct imaging of dynamical heterogeneities near the colloid-gel transition. Phys. Rev. Lett. 2007, 99, 078301.

(20) Dibble, C. J.; Kogan, M.; Solomon, M. J. Structural origins of dynamical heterogeneity in colloidal gels. Phys. Rev. E 2008, 77, 050401 .

(21) Rueb, C. J.; Zukoski, C. F. Viscoelastic Properties of Colloidal Gels. J. Rheol. 1997, 41, 197-218.

(22) Krall, A. H.; Weitz, D. A. Internal Dynamics and Elasticity of Fractal Colloidal Gels. Phys. Rev. Lett. 1998, 80, 778-781.

(23) Lindström, S. B.; Kodger, T. E.; Sprakel, J.; Weitz, D. A. Structures, Stresses, and Fluctuations in the Delayed Failure of Colloidal Gels. Soft Matter 2012, 8, 3657-3664.

(24) van Doorn, J. M.; Bronkhorst, J.; Higler, R.; Van De Laar, T.; Sprakel, J. Linking Particle Dynamics to Local Connectivity in Colloidal Gels. Phys. Rev. Lett. 2017, 118, 1-5.

(25) Srivastava, S.; Schaefer, J. L.; Yang, Z.; Tu, Z.; Archer, L. A. 25th Anniversary Article: Polymer-particle Composites: Phase Stability and Applications in Electrochemical Energy Storage. Adv. Mater. 2014, 26, 201-234.

(26) Anderson, B. J.; Zukoski, C. F. Rheology and Microstructure of Entangled Polymer Nanocomposite Melts. Macromolecules 2009, 42, $8370-8384$ 
(27) Kalathi, J. T.; Grest, G. S.; Kumar, S. K. Universal Viscosity Behavior of Polymer Nanocomposites. Phys. Rev. Lett. 2012, 109, 15.

(28) Meng, G.; Arkus, N.; Brenner, M. P.; Manoharan, V. N. The Free-energy Landscape of Clusters of Attractive Hard Spheres. Science 2010, 327, 560-563.

(29) Foffi, G.; Sciortino, F.; Tartaglia, P.; Zaccarelli, E.; Verso, F. L.; Reatto, L.; Dawson, K. A.; Likos, C. N. Structural Arrest in Dense Star-polymer Solutions. Phys. Rev. Lett. 2003, 90, 4.

(30) Stellbrink, J.; Allgaier, J.; Richter, D. Dynamics of Star Polymers: Evidence for a Structural Glass Transition. Phys. Rev. E: Stat. Phys., Plasmas, Fluids, Relat. Interdiscip. Top. 1997, 56, R3772R3775.

(31) Mayer, C.; Zaccarelli, E.; Stiakakis, E.; Likos, C. N.; Sciortino, F.; Munam, A.; Gauthier, M.; Hadjichristidis, N.; Iatrou, H.; Tartaglia, P.; Löwen, H.; Vlassopoulos, D. Asymmetric caging in soft colloidal mixtures. Nat. Mater. 2008, 7, 780-784.

(32) Marzi, D.; Capone, B.; Marakis, J.; Merola, M. C.; Truzzolillo, D.; Cipelletti, L.; Moingeon, F.; Gauthier, M.; Vlassopoulos, D.; Likos, C. N.; Camargo, M. Depletion, melting and reentrant solidification in mixtures of soft and hard colloids. Soft Matter 2015, 11, 8296-8312.

(33) Merola, M. C.; Parisi, D.; Truzzolillo, D.; Vlassopoulos, D.; Deepak, V. D.; Gauthier, M. Asymmetric soft-hard colloidal mixtures: Osmotic effects, glassy states and rheology. J. Rheol. 2018, 62, 63-79.

(34) Becher, P. Encyclopedia of Emulsion Technology, 1998; Vol. 1, p 57.

(35) Hunter, R. J. Foundations of Colloid Science; Clarendon: Oxford, 1989; Vol. II, pp 992-1052.

(36) Yeung, A.; Moran, K.; Masliyah, J.; Czarnecki, J. Shear-induced Coalescence of Emulsified Oil Drops. J. Colloid Interface Sci. 2003, 265, 439-443.

(37) Saiki, Y.; Prestidge, C. A.; Horn, R. G. Effects of Droplet Deformability on Emulsion Rheology. Colloids Surf., A 2007, 299, 65-72.

(38) Crocker, J. C.; Grier, D. G. Methods of Digital Video Microscopy for Colloidal Studies. J. Colloid Interface Sci. 1996, 179, 298-310.

(39) Gao, Y.; Kilfoil, M. L. Accurate Detection and Complete Tracking of Large Populations of Features in Three Dimensions. Opt. Express 2009, 17, 4685-4704.

(40) https://www.brenntag.com/media/documents/bsi/product_ data_sheets/material_science/ashland_polymers/pvp_polymers brochure.pdf (accessē October 2019).

(41) Rahman, A. Correlations in the Motion of Atoms in Liquid Argon. Phys. Rev. 1964, 136, A405.

(42) Chaudhuri, P.; Berthier, L.; Kob, W. Universal Nature of Particle Displacements Close to Glass and Jamming Transitions. Phys. Rev. Lett. 2007, 99, 2-5.

(43) van Blaaderen, A.; Wiltzius, P. Real-space Structure of Colloidal Hard-sphere Glasses. Science 1995, 270, 1177-1179.

(44) Weeks, E. R.; Crocker, J. C.; Levitt, A. C.; Schofield, A.; Weitz, D. A. Three-dimensional Direct Imaging of Structural Relaxation near the Colloidal Glass Transition. Science 2000, 287, 627-631.

(45) Cipelletti, L.; Ramos, L. Slow Dynamics in Glassy Soft Matter. J. Phys.: Condens. Matter 2005, 17, R253.

(46) Loiseau, J.; Doërr, N.; Suau, J. M.; Egraz, J. B.; Llauro, M. F.; Ladavière, C.; Claverie, J. Synthesis and Characterization of Poly(acrylic acid) Produced by RAFT Polymerization. Application as a very Efficient Dispersant of $\mathrm{CaCO} 3$, Kaolin, and $\mathrm{TiO}$. Macromolecules 2003, 36, 3066-3077.

(47) Llauro, M.-F.; Loiseau, J.; Boisson, F.; Delolme, F.; Ladavière, C.; Claverie, J. Unexpected End-groups of Poly(acrylic acid) Prepared by RAFT Polymerization. J. Polym. Sci., Part A: Polym. Chem. 2004, $42,5439-5462$

(48) Maniego, A. R.; Ang, D.; Guillaneuf, Y.; Lefay, C.; Gigmes, D.; Aldrich-Wright, J. R.; Gaborieau, M.; Castignolles, P. Separation of Poly(acrylic acid) Salts According to Topology Using Capillary
Electrophoresis in the Critical Conditions. Anal. Bioanal. Chem. 2013 405, 9009-9020.

(49) Lena, J.-B.; Goroncy, A. K.; Thevarajah, J. J.; Maniego, A. R.; Russell, G. T.; Castignolles, P.; Gaborieau, M. Effect of Transfer Agent, Temperature and Initial Monomer Concentration on Branching in Poly(acrylic acid): A Study by13C NMR Spectroscopy and Capillary Electrophoresis. Polymer 2017, 114, 209-220.

(50) Wang, D.; Dimonie, V. L.; Sudol, E. D.; El-Aasser, M. S. Effect of PVP in Dispersion and Seeded Dispersion Polymerizations. J. Appl. Polym. Sci. 2002, 84, 2721-2732.

(51) LeBel, R. G.; Goring, D. A. I. Density, Viscosity, Refractive Index, and Hygroscopicity of Mixtures of Water and Dimethyl Sulfoxide. J. Chem. Eng. Data 1962, 7, 100-101. 